\title{
How to define the therapeutic limits
}

\author{
Alberto Cester', Monica Maselli2, Francesco Bolzetta2 \\ 1 Chief of Medical Department and Geriatric Unit, UOC Geriatria Azienda ULL13 Mirano (VE), \\ Sede ospedaliera di Dolo \\ 2 Medical Department, Geriatric Unit, UOC Geriatria Azienda ULL13 Mirano (VE), Sede ospedaliera di Dolo, \\ Italy
}

\begin{abstract}
With the advance of technological progress and the increase in life expectancy, it is nowadays mandatory to define what is the therapeutic limit. Every day each physician must take therapeutic decisions on the basis of his scientific knowledge, but also of his own conscience and sense of limits. They can not avoid to consider the global risk of death, disability and morbidity in more advanced age, especially in the field of cardiology and cardiac surgery. In these subjects, both fit that frail, is necessary not only an evaluation for adequate risk assessment, but also a multidimensional assessment performed with advanced tools.

The resilience of the subject, ability for which some patients considered out of therapy demonstrate the ability to adapt and overcome critical phases, must also be weighed. Where and what is the therapeutic limit should be evaluated individually with humility, competence and capacity for dialogue with other disciplines in a work team that respects the individual professionalism.

In recent years, much has changed in the cardiology/cardiac surgery approach in old people. With the development of less or minimal invasive new techniques, there are no more insurmountable limits that can be connected only to the chronological age of the patients.
\end{abstract}

Health promotion and prevention in the elderly aim to reduce premature mortality for acute and chronic diseases, to preserve as long as possible patient's functional independence, increase life expectancy, and increase the quality of life, even in the last decades.

Corresponding author: Alberto Cester, Distretto Dolo-Mirano Azienda ULSS3 “Serenissima” Mestre (VE), Riviera XXIX Aprile 2, 30031 Dolo (VE), Italy. E-mail: alberto.cester@aulss3.veneto.it

Key words: Frailty; frail; elderly, ageing; cardiovascular diseases.

Received for publication: 25 November 2016

Accepted for publication: 5 December 2016

(C) Copyright A. Cester et al., 2017

Tipografia PI-ME Editrice, Italy

Monaldi Archives for Chest Disease 2017; 87:846

doi: 10.4081/monaldi.2017.846

This article is distributed under the terms of the Creative Commons Attribution Noncommercial License (by-nc 4.0) which permits any noncommercial use, distribution, and reproduction in any medium provided the original author(s) and source are credited.
Chronic diseases are typical of the elderly patient; there is nothing more unstable than the so-called chronicity both from a clinical standpoint and from a social point of view. However, chronicity is not synonymous with weakness.

Frailty is a new and emerging syndrome in the field of geriatrics. It is a physiological condition, but a reliable marker or a precise definition has not been identified yet. The definition that we prefer is the one of Fretwell: frailty as marked vulnerability and decreased homeostatic ability. If it is an open question whether frail, clinical complexity and cascade pathologies exist or are just intrinsic aging factors. The fact that frailty is not present in every elderly suggests that it is associated with aging but that it is not an inevitable process, and so it may be prevented or treated.

The study of frailty may provide an explanation for the downward spiral of many elderly patients after an acute illness and hospitalization [1]. Frail individuals have greater nonspecific vulnerability and are more susceptible to adverse health outcomes, including hospitalization and mortality [2].

The complexity of frail patient requires an adequate assessment methodology, which needs to be multidimensional with an integrated network of hospital and territorial structures. The multidimensional assessment includes collaboration between several professional figures gathered in a team, in which organizational and cultural presumptions, suspiciousness, contentiousness, jealousy management, preconceived closures to other experiences are eliminated and essential aspects of others' skills are acquired. In this context also the continuous communication and interaction with caregivers and the general practitioner are mandatory. The aim is to identify a tailored assistance program for each patient.

How to recognize and measure the frailty? Frailty can be given by a reduced muscle capacity, reduced physical endurance, decreased ability to feed itself and reduced neuropsychological abilities. The frail patient is typically a person $>75$ years old, with important morbidity, that assume many drugs and experience frequently serious impairment in cognitive, functional and social status. One of the most accepted diagnostic criteria is that proposed by Linda Fried [2], a biological model, that define as frail a patient with 3 or more of the following (when there are fewer than three items we talk about pre-frail individual): unintentional weight loss (10 pounds in the last year), presence of exhaustion (selfreported fatigue in at least 3 days / week), weak grip strength (less than 13 and 7.5 pounds in males and females, respectively), slow walking (more than $7 \mathrm{sec}$. to walk 4.57 meters) or low physical activity (assessed using PASE-Physical Activity Scale for the Elderly). Moreover, also functional models with asses of physical, nutritional, cognitive and sensory has been proposed [3] as well as cumulative deficit models [4]. Beyond various definition, a correct approach to the frail patient needs clinical and logical ability, and also a great adaptability to the sudden changes that typically affect these patients, always keeping in mind as final goal to do what is best for the person himself. 
For an appropriate assessment of the frail patients, especially when we have to take treatment decisions, there is also a need of clinical evaluation scales. In hospitalized elderly one of the possible geriatric tools to evaluate the risk ratio is the Multidimensional Prognostic Index (MPI). The MPI, calculated from information collected in a standardized comprehensive geriatric assessment (CGA) combining multiple functional and cognitive scale as well as drugs intake and degree of morbidity, accurately stratifies hospitalized elderly patients into groups at varying risk of mortality. It represents a reliable and sensitive measurement of risk assessment that might be useful for clinical and research purposes [5]. This is true also to predict mortality at shortterm in elderly patients with heart failure [6].

Similarly, in patients with advanced dementia there are issues about the usefulness of artificial nutrition in end stage disease [7]. In fact, in seven studies in patients with severe dementia, no significant association between artificial nutrition and nutritional improvement, reduced incidence of pressure ulcers and increased survival has been observed. One study, moreover, showed an increased risk of aspiration pneumonia and an increased use of body restraints [8]. Artificial feeding also appears to reduce the contact with the caregiver and the pleasure of food taste [9]. Also in this patients there are useful tools to perform a prognostic evaluation such as the Clinical Dementia Rating Scale (CDR 5), which assesses the need to total care, the inability to communicate, bedridden, double incontinence [10], and the Functional Assessment Stages (FAST 7C), that evaluate the presence of severe dementia with complete dependence, limited vocabulary of one word or no, double incontinence, inability to walk [11]. Additional criteria that increase the prognostic predictability are: weight loss $>10 \%$ in 6 months, hypoalbuminemia, the presence of pressure sores ( $3^{\text {rd }}$ or $4^{\text {th }}$ stage), recurrent fever and pneumonia.

When we have to identify and determine where the therapeutic limit is, an adequate elderly patient assessment can not be separated from the analysis of his resilience. Resilience [from lat. resiliens,-lientis, pres. part. of resilire "jump back, bouncing"] is the ability to withstand sudden impacts without breaking. In engineering resilience is the ability of a material to resist breaking forces; in computer science, resilience is the ability of a system to adapt to the conditions of use and resist wear; in ecology and biology resilience is the ability to repair itself after damage. In psychology, resilience is seen as a man's ability to face and overcome life's adversities. De Alfieri in a recent paper explained how shifting research from a negative concern (frailty) to a positive one (resilience) might achieve relevant effects, also because restoring a system to its previous state can be complex, expensive, and sometimes even impossible, whereas a resilient system can withstand shocks and rebuild itself when necessary. Resilience is the added capacity of humans to anticipate and plan for the future. At any age [12].

Frailty has an important prognostic value also in cardiovascular disease, that are nowadays the leading cause of death. For example, frailty has been proved to be independently associated with 1 year mortality in elderly patients with non ST-segment elevation myocardial infarction [13]. Moreover, cardiovascular diseases are one of the most frequent causes of morbidity in the elderly, especially between frail ones. The topic is so interesting in the last years that the Italian Heart Journal, the official journal of the Italian Federation of Cardiology and the Italian Society for Cardiac Surgery, dedicated a whole supplement to the problem of heart failure in the old people. Heart failure is in fact a typical disease of old age and represents one of the major causes of disability. The relevance and the need of prognostic indexes to evaluate overall risk in elderly patients undergoing cardiovascular surgery are increasing, also for the progressive increasing in life expectancy. In order to simplify and make the evaluation of the risk and stratification faster in old patients with heart failure others tools have been developed, such the evaluation from the EFFECT study [14], that combines 11 variables (age, sex, blood pressure, respiratory rate, some specific comorbidities and laboratory tests) and from the ADHERE study, that considers only 4 simple parameters (systolic blood pressure, heart rate, BUN levels and age) [15].

In this perspective a comprehensive geriatric assessment with risk stratification become fundamental to avoid attitudes that would lengthen the life span without ensuring a residual good quality of life. Once again, the role of a geriatric approach to cardiovascular diseases is first of all to prioritizing the goals, identifying and distinguishing the fit elderly from the frail ones. This is important in order to plan a tailored program for each patient, that privileges life-threatening interventions for fit people and interventions to preserve the functionality, or only to relieve suffering, in frail ones. There is also a strong need to elucidate the role of health care network to manage the end-stage of cardiovascular diseases

\section{References}

1. Ahmed N, Mandel R, Fain MJ. Frailty: an emerging geriatric syndrome. Am J Med 2007;120:748-53.

2. Fried, Waltson, Ferrucci, Hazzard's Geriatric Medicine and Gerontology, 6th Edition, 2009: 631-45.

3. Strawbridge WJ, Shema SJ, Balfour JL, et al. Antecedents of frailty over three decades in an older cohort. J Gerontol B Psychol Sci Soc Sci 1998;53:S9-16.

4. Rockwood K, Song X, MacKnight C, et al. A global clinical measure of fitness and frailty in elderly people. CMAJ 2005;173:489-95.

5. Pilotto A, Ferrucci L, Franceschi M, et al. Development and validation of a multidimensional prognostic index for one-year mortality from comprehensive geriatric assessment in hospitalized older patients. Rejuvenation Res 2008;11:151-61.

6. Pilotto A, Addante F, Franceschi M, et al. Multidimensional Prognostic Index based on a comprehensive geriatric assessment predicts short-term mortality in older patients with heart failure. Circ Heart Fail 2010;3:14-20.

7. Gruppo di studio la cura nella fase terminale della malattia. Le cure di fine vita in Geriatria. Novembre 2008.

8. Sampson EL, Candy B, Jones L. Enteral tube feeding for older people with advanced dementia. Cochrane Database Syst Rev 2009; CD007209.

9. Volicer L. Goals of care in advanced dementia: quality of life, dignity and comfort. J Nutr Health Aging 2007;11:481.

10. Hughes CP, Berg L, Danziger WL, et al. A new clinical scale for the staging of dementia.Br J Psychiatry 1982;140:566-72.

11. Reisberg B Alzheimer's disease. Stages of cognitive decline. Am J Nurs 1984;84:225-8.

12. De Alfieri W, Borgogni T. Through the looking glass and what frailty found there: looking for resilience in older adults. J Am Geriatr Soc 2010;58:602-3.

13. Ekerstad N, Swahn E, Janzon M, et al. Frailty is independently associated with 1-year mortality for elderly patients with non-ST-segment elevation myocardial infarction. Eur J Prev Cardiol 2014;21: 1216-24.

14. Lee DS, Austin PC, Rouleau JL, et al.Predicting mortality among patients hospitalized for heart failure: derivation and validation of a clinical model. JAMA 2003;290:2581-7.

15. Fonarow GC, Adams KF Jr, Abraham WT, et al. Risk stratification for in-hospital mortality in acutely decompensated heart failure: classification and regression tree analysis. JAMA 2005;293:572-80. 